\title{
Digital Building Twins - Contributions of the ANR McBIM Project
}

\author{
Ana Roxin \\ LIB EA 7534 \\ Univ. Bourgogne Franche-Comté \\ Dijon, France \\ 0000-0001-9841-0494
}

\author{
William Derigent \\ CRAN CNRS UMR 7039 \\ Université de Lorraine \\ Nancy, France \\ william.derigent@univ-lorraine.fr
}

\author{
Wahabou Abdou \\ LIB EA 7534 \\ Univ. Bourgogne Franche-Comté \\ Dijon, France \\ wahabou.abdou@u-bourgogne.fr
}

\author{
Daniela Dragomirescu \\ $L A A S$ \\ National Institute of Applied Sciences \\ Toulouse, France \\ daniela@laas.fr
}

\author{
Dominique Ginhac \\ ImViA EA 7535 \\ Univ. Bourgogne Franche-Comté \\ Dijon, France \\ dominique.ginhac@u-bourgogne.fr
Laurent Montegut
360 SmartConnect \\ FINAO SAS \\ Trans en Provence, France \\ lm@360sc.io
}

\begin{abstract}
This paper presents the vision behind the McBIM project, funded by the French National Agency of Research (ANR). McBIM stands for "Communicating Matter for BIM" and is a concept coined by the CRAN laboratory, concept at the basis of the scientific contributions of the project. Building upon latest advances in Semantic Web technologies, Wireless Sensor Networks and digital twins in AEC (Architecture, Engineering and Construction), the project aims at delivering real-time integration of sensor data into accurate digital building representations. The overall goal is to enable actionable knowledge, meaning knowledge that supports decision making. After a brief summary of the issues addressed and the overall project vision, this article depicts main contributions made so far in the context of the ANR McBIM project. These address semantic interoperability with BIM standards (e.g. IFC ISO 16739), routing in wireless sensor networks along with autonomic computing.

Index Terms-IFC, Semantic Web, Linked Data, Property Sets, Semantic Interoperability, Wireless Sensor Networks, Autonomic Computing, Building Information Modeling, Building Digital Twin, Concrete
\end{abstract}

\section{INTRODUCTION}

The complexity of information required for todays decisions for the public sector, the private sector and for the citizens, requires digital techniques. Consequently, there are huge investments in digitization at the national, regional and global levels, and a need for collaboration and interoperability between systems. Digital techniques do not ensure this to happen by themselves. The observed environment has traditionally been modelled using techniques from the GIS domain, while the built environment is modelled using BIM techniques. The traditional difference in granularity between these two, GIS and BIM, perspectives is more and more vanishing. Use cases and perspectives converge and largely overlaps. The models from the two domains are tightly bound to each other, every built construction has a location in the existing environment, the Earth (as long as we do not consider outer space), and GIS incorporates all built environments. Each of these two domains spend huge investments in capturing information from the other domain today, mostly resulting in double spending. The removal of the barriers to share information across the domain barriers will not only save large investments, but also result in information of higher quality. There is an increasing need for projects to move seamlessly between planning and civil infrastructure design (GIS-and civil CAD-based work) to design, build, and operate (BIM based work).

\section{OVERVIEW AND GENERAL RESEARCH ISSUES FOR THE ANR MCBIM PROJECT}

\section{A. Communicating materials}

The concept of communicating materials was coined by the Research Centre for Automatic Control (CRAN) back in 2009. This concept encompasses the idea of materials that can communicate with their environment, process, exchange information, and store data in their own structure. Such communicating materials also have the capacity to sense their environment and measure their own internal physical states. When applied to the construction industry, the concept of communicating materials led to a physical prototype based on RFID tags embedded into the material. Still, with time, the drawbacks and limitations of RFID pushed researchers to look for alternative approaches. Moreover, given the rise of BIM (Building Information Modeling) as an application domain, it became pertaining to seek efficient integration of communicating materials directly into buildings. Indeed, this would allow addressing existing BIM limitations notably the necessity to rebuild a buildings model for each of its lifecycle phases. With the current trend of digital building twins, as accurate realtime digital representations of buildings or infrastructures, it becomes even more urgent to make constructions aware of their environment and their state. 


\section{ThE ANR MCBIM (COMMUNICATING MATERIALS FOR BIM) PROJECT}

With the above issues in mind, the McBIM project has recently been selected for funding by the French National Research Agency (ANR). Bringing together a consortium of academics and industrials, the goals of the project are to:

- design a communicating concrete meaning embed concrete building elements with sensors,

- integrate the sensed data into BIM platforms,

- prove the usefulness of this approach across two building lifecycle phases, namely construction and exploitation phases (for structural health monitoring).

To build this communicating concrete, several research issues must be addressed:

- design robust wireless communications, not impacted by the concrete environment,

- implement innovative RF harvesting techniques to maximize the lifetime of embedded sensor nodes,

- specify new data management strategies controlling how data (either generated by sensor nodes, or sent by users) is spread into the WSN for a fast and reliable data storage and retrieval,

- address interoperability issues, basing building description on the IFC standard,

- allow obtaining actionable knowledge from sensor data,

- ensure ISO 19650 [1]

Given their multi-disciplinary nature, the researches pursued in the context of the McBIM project clearly address the central issue of delivering efficient and interoperable digital building twins. As mentioned in the introduction, this is a different concept from BIM, notably from the point of view of the goals pursued. Indeed, while BIM only renders the elements contained in an IFC file, a digital building twin must integrate real-time data, mainly obtained from sensors integrated into the building.

The sections below further explain the existing approaches related to the issues listed above and depict how the McBIM will address those issues.

\section{SCIENTIFIC BACKGROUND}

Before describing the research orientations took in the context of the ANR McBIM project, this section will resume main existing standards pertaining to the issues considered.

\section{A. Energy efficiency in WSN}

Energy efficiency is a very important issue in wireless sensor networks. Sensors generally have limited energy. One way to reduce energy consumption could be to reduce the transmission power. However, this method reduces the transmission range and leads to increase the number of hops. Moreover, in an environment where the radio signal attenuation could be important, reducing the transmission power may prevent wide dissemination of messages. Another approach to harvest sensors' energy is to arrange them so that they do not remain active during the hole monitoring period. The sensors will alternate the active and inactive (sleep) modes. It is then crucial to ensure the availability of routes to transmit any messages when a node wakes up. The mechanisms which reduce the energy consumption in wireless sensor networks can be classified into three groups:

- those that rely on the message content

- those based on mobility

- those which use duty cycling

Content-based approaches attempt to ensure that only useful data is transmitted over the network. Data can be processed locally (on each node or within each cluster). Thus, the processes are not transferred only at the receiver (generally the sink). Content-based methods use data aggregation, compression, and forecasts.

Energy savings achieved through mobility schemes allow (if possible) to spread the communication load that may be related to the geographical location of the nodes. For example, the nodes located close to the sink are very likely to be requested to route messages towards the sink. Therefore, their energy consumption might be high, compared to the rest of the network. This group of protocols allows node movements according to the physical topology of the network.

Duty cycling is an effective energy conservation mechanism in sensor networks. It allows to alternate active and sleep periods. The lower the duty cycle, the longer a node is idle and saves energy. This lengthens the network lifetime. The duty cycle can be applied to all subsystems of a sensor including the radio communication subsystem. When they are actives, nodes can send or receive messages, or simply listen to the radio channel. The inactive listening (idle) can represent a significant consumption of energy over time. Duty cycling can be implemented across multiple layers or across multiple layers, including data link, network, and transport layers. However, for these mechanisms, one must remain watchful on the impact of the temporary unavailability of some nodes. This could affect both network coverage and loss of connectivity.

The duty cycling is favored by two common phenomena. First, it exploits the redundancy in wireless sensor deployment [2]. The system can thus adaptively select only a minimal subset of nodes that will remain temporarily active to maintain connectivity. Second, it exploits the fact that in most applications, occurrences of events are rare. The nodes spend the majority of their time listening to the channel. This means that even the selected subset of nodes does not need to be activated all the time.

\section{B. Data dissemination algorithms for WSN}

A wireless sensor network is a collection of sensor nodes communicating through wireless connections. Since every node has a limited coverage area, to widely spread data over the network, a multi-hop communication protocol is required. This means nodes should cooperate and intermediate nodes should accept to relay packets. In such a network, each node acts simultaneously as a client node and as a wireless router, allowing multi-hop packet forwarding. Routing is the process of finding a path to send data between two (or more) nodes. 


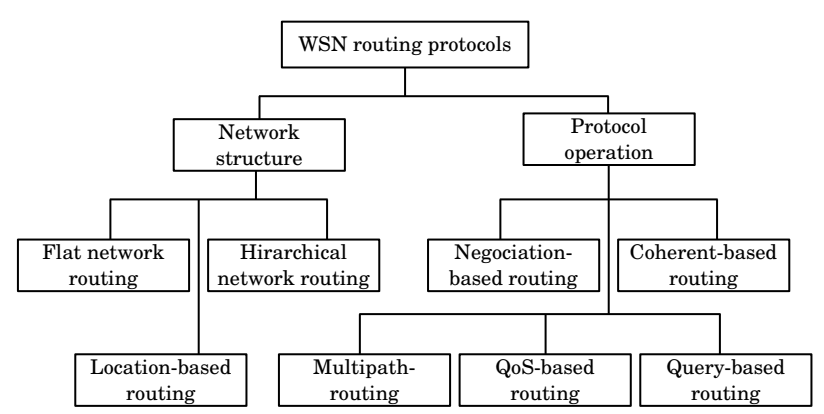

Fig. 1. classification of WSN routing protocols [6]

In literature, a lot of routing protocols dedicated to WSN have been proposed. Various classifications of routing protocols have been done [3]-[5].

The routing process could depend on the network structure (flat, hierarchical, location-based). Flat routing protocols (flooding, gossiping, data centric) are used when every node plays the same role. In hierarchical routing protocols, nodes play different roles according to their position, thier neighborhood and/or their energy. This allows to cope with scalability.

LEACH (Low Energy Adaptive Clustering Hierarchy) [7] is one of the most popular WSN clustering protocol. It computes, in a distributed way, the clusters. The cluster heads $(\mathrm{CH})$ are selected based on a probability $p$, and each node selects the $\mathrm{CH}$ with whom it can communicate with the minimum energy. The $\mathrm{CH}$ role change over time in order to allot the traffic load and energy consumption. PEGASIS (Power-Efficient GAthering in Sensor Information Systems) [8] is proposed to improve LEACH by forming a chain among nodes. Communications a favored between close neighbors.

The CCM (Chain-Cluster based Mixed routing) [9] protocol take advantages on both LEACH (latency) and PEGASIS (energy consumption). It relies on two steps: forming routing chains and voting for a head (among chain heads) which will send aggregated data to the base station or sink.

CRBCC (Chain Routing Based on Coordinates-oriented Cluster) [10] divides the network into cluster according to geographical coordinates. The clusters are formed based on the $\mathrm{Y}$ coordinate and the algorithm forms a chain within each cluster. Thereafter, every chain elects a leader.

Balanced Chain-Based Routing Protocol (BCBRP) [11] is another data dissemination protocol that aims to extend the lifespan of the sensor nodes. It relies on three steps: (i) split the network into several subnetworks with equal size, then (ii) select a bridge node within each subnetwork and finally (iii) build a chain which interconnect the subnetworks via the bridges. Table I summarizes the benefits of the protocols.

\section{BIM Standards}

Among the most-known definitions of BIM, we may cite the one from Eastman et al.: BIM is a technology that allows one or more accurate virtual models of a building to be

\begin{tabular}{|l|c|c|c|c|}
\hline $\begin{array}{l}\text { Protocol } \\
\text { name }\end{array}$ & $\begin{array}{l}\text { Energy ef- } \\
\text { ficiency }\end{array}$ & $\begin{array}{l}\text { Maintaining } \\
\text { cost }\end{array}$ & $\begin{array}{l}\text { Resilience } \\
\text { to link } \\
\text { failure }\end{array}$ & scalability \\
\hline LEACH & -- & + & + & -- \\
\hline PEGASIS & - & -- & - & -- \\
\hline CCM & + & - & - & -- \\
\hline CRBCC & + & + & + & - \\
\hline BCBRP & - & -- & ++ & -- \\
\hline
\end{tabular}

TABLE I

COMPARISON OF DATA DISSEMINATION PROTOCOLS [12]

constructed digitally. They support design through its phases, allowing better analysis and control than manual processes. When completed, these computer-generated models contain precise geometry and data needed to support the construction, fabrication, and procurement activities through which the building is realized. ISO 29481 or BIM Information Delivery Manual Part1: Methodology and format ( [13]) defines BIM as a shared digital representation of an object built to facilitate design, construction and operating process and form a reliable basis for decision-making. From this definition, BIM is more focused on managing business processes as done during the whole lifecycle of the building, than delivering a real-time 3D representation of the state of a building.

In the context of the ANR McBIM project, BIM must allow generating, storing and exchanging information about building elements and sensors in an interoperable and reusable way. Therefore, in our vision, a BIM-based model goes beyond a simple three-dimensional model of a building that solely includes geometrical data such as models conceived with computer-aided design (CAD)-based tools. This is because a BIM model also needs to include semantic information. How this can be achieved is discussed below in section IV-E. The scope of this section is to briefly present the main standards forming the building bricks of BIM. These standards are the following:

- Processes are described following ISO 29481:2016 or Information Delivery Manual [13]. IDM is an approach for defining Exchange Requirements (ER) in natural language and mainly addresses the planning lifecycle. It comprises a specification of what kind of information must a contracted exchange hold. IDMs are defined by domain experts (or BIM users) and software developers (or BIM providers). Processes are specified using Business Process Model and Notation (BPMN) and usually include stakeholders, project stages and activities.

- Building data is expressed by means of the open standard defined in ISO 16739:2018 and called Industrial Foundation Classes [14]. IFC represents a buildings conceptual model and is specified in EXPRESS, while being fully compliant with STEP (Standard for the Exchange of Product data) as defined in ISO 10303 ( [15]). STEP defines a modular architecture relying on the STEPMOD development and publication framework, also specified in EXPRESS. IFC is an open format, public and nonproprietary, promoted by the international standardization 
organization bSI (buildingSMART International). The current version of IFC is IFC4.1, but IFC4.2 should be made official during 2019.

- Model-View Definitions (MVD) allow selecting only the specific parts of the IFC Schema as required for meeting the ERs defined in the IDM. An MVD is a subset of the full IFC Schema (ISO 29481-3). MVDs are specified using mvdXML and are used for compliance and conformity-checking over IFC files.

- Terms are defined by means of dictionaries, following the International Framework for Dictionaries (IFD) or ISO 12006-3 [16]. While one would suspect that terms are semantically defined, the approach implemented today for the bSDD (buildingSMART Data Dictionary) is still keyword-based. Several projects have delivered semantic versions of bSDD e.g. bSDDowl [17] but none of their results has yet been officially implemented at the level of bSI.

More specifically, the next paragraphs will further specify IFC definitions as pertaining to building elements that can be made from concrete. Before this, a few general considerations will help reaching a better understanding of how these elements are structured inside the IFC Schema. IFC considers building elements (IfcElement) as generalizations of all components composing an AEC product. Walls or windows are typical examples of building elements. The IfcRelContainedInSpatialStructure allows linking an instance of the IfcElement class to a spatial structure considered in the project e.g. site, building or storey. An element has a particular element type, instantiated through the IfcRelDefinesByType property. The IfcElement class has several sub-classes, out of which only two pertain to building elements made of concrete. These are IfcBuildingElement and IfcCivilElement. While the latter is to be used for elements in civil engineering works that cannot be represented as IfcBuildingElements, the first comprises an exhaustive list of tangible elements that define a buildings structural and space separating system.

Among those elements (sub-classes of the IfcBuildingElement class), the table below lists the ones pertaining in the context of the ANR McBIM project. For each class listed, the specific Property Sets (Psets) as defined in the IFC schema are also listed. In addition to those Property Sets, each class mentioned in Table II also implements the 3 Psets for concrete or precast concrete elements, namely Pset_ConcreteElementGeneral, Pset_PrecastConcreteElementFabrication

and Pset_PrecastConcreteElementGeneral.

The IFC specification considers property sets as containers for property hierarchies or property trees. Properties missing from the existing Psets defined, can be defined by users. The only constraint imposed by the IFC standard is to remove the Pset_ prefix from the names of property sets not included in the standard.

\begin{tabular}{|l|l|}
\hline \multirow{2}{*}{ IfcBeam } & IFC Property Sets \\
\hline IfcBuildingProxyElement & Pset_BeamCommon \\
\cline { 2 - 2 } & Pset_ReinforcementBarPitchOfBeam \\
\hline IfcChimney & Pset_BuildingProxyCommon \\
\cline { 2 - 2 } & Pset_ChimneyCommon \\
\hline \multirow{2}{*}{ IfcColumn } & Pset_ReinforcementBarPitchOfColumn \\
\hline IfcCurtainWall & Pset_ColumnCommon \\
\cline { 2 - 2 } IfcFooting & Pset_ReinforcementBarPitchOfColumn \\
\hline IfcMember & Pset_CurtainWallCommon \\
\hline IfcPlate & Pset_FootingCommon \\
\hline IfcRailing & Pset_MemberCommon \\
\hline IfcRamp & Pset_PlateCommon \\
\hline IfcRampFlight & Pset_RailingCommon \\
\hline IfcRoof & Pset_RampCommon \\
\hline IfcSlab & Pset_RampFlightCommon \\
\hline \multirow{5}{*}{} & Pset_RoofCommon \\
\hline IfcStair & Pset_SlabCommon \\
\cline { 2 - 2 } & Pset_PrecastSlab \\
\cline { 2 - 2 } IfcStairFlight & Pset_ReinforcementBarPitchOfSlab \\
\hline IfcWall & Pset_StairCommon \\
\hline & Pset_StairFlightCommon \\
\cline { 2 - 2 } & Pset_WallCommon \\
\cline { 2 - 2 } & Pset_ReinforcementBarPitchOfWall \\
\hline
\end{tabular}

TABLE II

IFCBUILDINGELEMENTS MADE OF CONCRETE

\section{Achieving interoperability}

In order to address this, we need to take a step back and discuss what is interoperability. Several definitions existing for this term. Even at the level of international standardization organizations such as ISO, there is no consensus regarding what interoperability means. The concept is generally defined in the context of a domain of knowledge or application. ISO 21127:2014 defines interoperability as implying that either two systems can exchange information or that multiple systems can be accessed with a single method [18]. Several types of interoperability are considered:

- Physical interoperability concerns the computation, use, transfer and exchange of data, as defined in ISO/IEC 20944-1:2013 [19]

- Syntactic interoperability is defined in ISO 16678:2014 as the ability of two or more systems or services to exchange structured information [20]

- Semantic interoperability, as defined in ISO/IEC 19941:2017, enables the meaning of the data model within the context of a subject area [to be] understood by the participating systems [21]

The above interoperability types are all connected to each other e.g. implementing an interoperability of Level L requires the implementation of an interoperability of the level (L-1). Semantic interoperability is the highest level of interoperability that can be reached. Achieving semantic interoperability cannot be done without reaching physical and syntactic interoperability. Hopefully, these two levels have already been fully addressed by existing and well-established computer science standards. Notably physical interoperability is reached through the usage of hardware standards (e.g. Ethernet) combined with standard network protocols (e.g. TCP/IP or HTTP). Syntactic interoperability has also been resolved through the 
specification and implementation of syntax standards such as XML, HTML, WSDL or SOAP.

When it comes to semantic interoperability, several flavours exist, each resulting in different types of actions that can be performed on the underlying knowledge. In order to best apprehend these concepts, we will start from existing ISO definitions for semantic interoperability:

- ISO 13606-1:2018 defines it as the ability for data shared by systems to be understood at the level of fully defined domain concepts [22]. This definition points to a first level of semantic interoperability which is understanding of data. Such understanding is usually characterized as minimum semantic interoperability. It is enabled by approaches based on Resource Description Framework (RDF). In this case, only the minimum knowledge is modelled: the concept of a building is related to the concept sensor through the relation contains.

- ISO 16678:2014 considers such interoperability as the ability of two or more systems or services to automatically interpret and use information that has been exchanged accurately [20]. This definition places the need for information interpretation, information being defined as contextualized data. This is called extended semantic interoperability and requires minimum semantic interoperability (thus RDF). This is called extended semantic interoperability and requires minimum semantic interoperability (thus RDF). The RDF Schema language allows defining a common interpretation of the elements contained in the message exchanged. Following our previous example, with RDF Schema the building concept is identified by an URI (Unified Resource Identifier) allowing a computer agent to dereference the concept and access an RDFS-defined ontology specifying additional knowledge about a building. Extended semantic interoperability allows obtaining additional knowledge about the concepts handled, but it does not allow to constraint such knowledge. Following our example, with this level of interoperability it is impossible to specify that if a sensor is in a building, the same sensor cannot be contained in a different building

- The definition from ISO/IEC 19941:2017 provided above, underlines the need for understanding meaning [21]. This is generally understood as full semantic interoperability and requires higher-order ontology description languages from the OWL family such as OWL-DL, OWL 2 RL, etc. This level of interoperability allows bounding knowledge: allowed interpretations represent the lower bound, while constraints preventing specific inferences form the upper bound. In the context of our building containing sensors, an incoherence would be notified if the same sensor instance is located in two different building instances.

Regarding the digitalization of construction, engineering and architecture (AEC), the French expert commission PTNB identified Linked Data and Semantic Web technologies as the only approach that fully addresses the issues related to interoperability in these domains. The next section will briefly present Semantic Web standards and main approaches pertaining for the considered application domain.

\section{E. Semantic Web technologies for BIM and IoT}

In the last decade, Semantic Web and Linked Data technologies have received increasingly more attention to facilitate knowledge modelling in the AEC/FM sector. In 2016, a Semantic Modelling and Linking Guide was issued at the international standards summit of bSI, in Barcelona. Since then, the topic of using semantics for delivering actionable knowledge has continuously gained attention from both researchers and industrials. The scope of this section is not to provide a state-of-the-art regarding Semantic Web, but to list and describe main conceptual differences associated to knowledge modelling with Semantic Web languages such as RDFS or OWL.

Indeed, modelling with Semantic Web languages is different than traditional modelling approaches. For example, in the EXPRESS language (used for serializing IFC), all the concepts are related to one single key or primary (meta-)concept. In EXPRESS, a property is always declared in the context of an entity. With Semantic Web languages, classes and properties are defined independently. The belonging of an instance to a class is determined by the set of necessary and sufficient conditions that the individual must observe. In the context of Semantic Web, a class is defined as an ensemble of properties its individuals must all implement, with specific values. Another difference of semantic modelling is the Open World Assumption (OWA), which states that it is not because some knowledge is missing or was not specified that it must be assumed as false. In EXPRESS, what is not specified is by default assumed false it is called a Closed World Assumption (CWA). Finally, another modelling difference relies in the No Unique Name Assumption (UNA) that is intrinsic to Semantic Web technologies. In Semantic Web, resources are identified by means of URIs (Unique Resource Identifiers). If two URIs are identical, then the resources they identify are also identical. In the context of BIM, resources are identified by means of so-called GUIDs (Globally Unique Identifiers) that usually contain UUID data. The main issue with this approach is that these GUIDs are not unique from one IFC file to the other. This is mainly justified by the freedom of implementation provided by the IFC standard for software companies.

\section{THE MCBIM VISION AND APPROACH PUSHING FORWARD THE STATE OF THE ART}

The McBIM project envisions contributions at various levels in smart buildings and communicating materials. One of his contributions will focus on the interoperability of models used in the description of knowledge in BIM. Then, in the field of communicating materials, the project will deal with several topics: (i) management of sensor network topology to ensure good coverage and efficient communications, (ii) autonomous sensor management and (iii) ) the security of the exchanges in the network. 


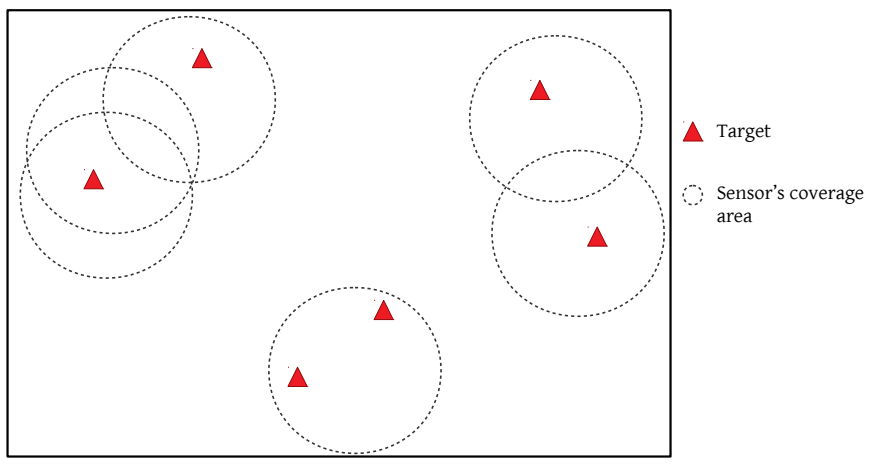

Fig. 2. Target Coverage

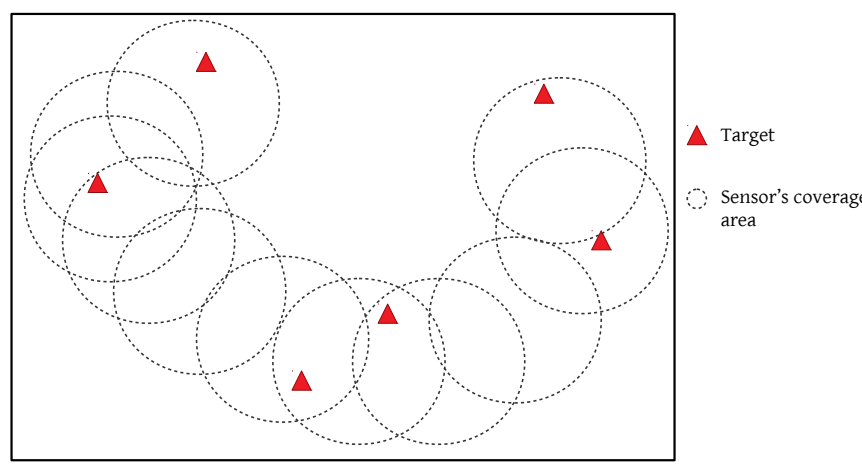

Fig. 3. Target Coverage while ensuring network connectivity

\section{A. Management of the topology in the sensor network}

The sensors will be poured into the concrete so that they can monitor specific areas of a wall. This means that the sensors should be placed around these locations. The places to monitor are called targets. In order to extend the network lifetime and have a fault-tolerant system, the sensors could be deployed in a redundant way. The sensor placement around specific areas is similar to the target monitoring problem [23].

In addition to the deployment of sensors around the targets (Figure 2), in order to form a connected network, some sensors would have to be placed in order to provide interconnection and act as relay nodes (Figure 3). The challenge amounts the maximization of sensors deployment around targets while minimizing (or avoiding) the risk of breaking the network connectivity. This is a multi-objective optimization problem. Two approaches can be used for modeling the problem. (i) A fixed number of sensors can be considered to optimize their placement. Another way of dealing with the problem is (ii) to determine the minimum number of sensors to monitor the targets and thereafter add a few nodes for connectivity purpose. To ensure the connectivity one can draw inspiration from the construction techniques of the Steiner tree. Our early works based on evolutionary algorithmic [24] and stochastic physicsbased optimization algorithm [25] have already yielded inter-

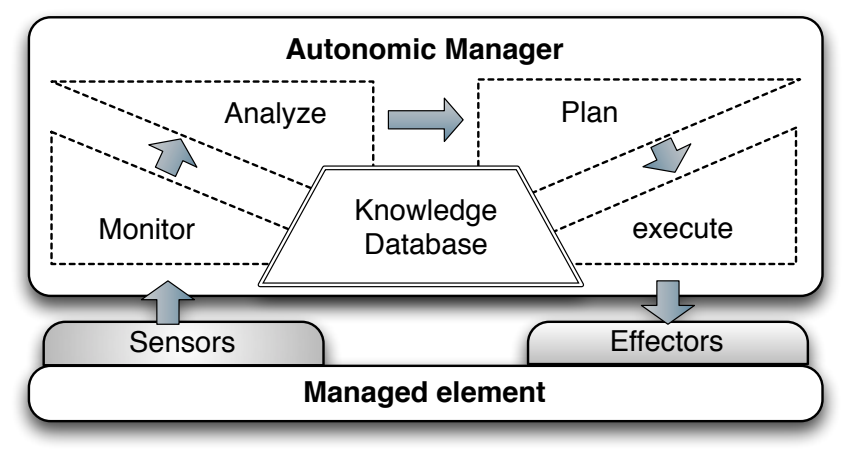

Fig. 4. Autonomous Element

esting results for target coverage. These approaches should now be adapted taking into account the specificities of the concrete environment.

\section{B. Autonomic computing}

When the sensors are poured into concrete, human intervention to manage them should be reduced at a bare minimum. Nodes should be able to operate autonomously. The autonomic computing paradigm allows attaining such level of system management.

Self-management systems with limited human interventions allow to cope with complex management systems and reduces the overall maintenance costs. Systems become a collection of interconnected autonomous entities. The autonomic computing paradigm was initially inspired by nature, especially the autonomic nervous system. The main objectives for autonomic systems are self-configuration, self-healing, self-optimization and self-protection (also known as self-chop). These objectives have been recently extended [26]. To ensure self-chop properties, autonomous systems must be able to interact with their environment thanks to sensor and effector modules. In addition they must have a knowledge base that can be made up of a simple configuration rules or enriched by artificial intelligence algorithms. The interaction with the environment must be continuous. It makes it possible to adapt to changing contexts. The overall working process of such a system is described by a closed control loop [27] (illustrated by Figure 4).

\section{CONCLUSION}

This paper presents contribution of the ANR McBIM project within the concept of digital building twins. This project focuses on the design of communicating concretes. The paper proposes a review of the state of the art on BIM standards and WSN communications. Thereafter it introduces some trails to efficiently deploy sensor nodes and to manage them in an autonomous way (thanks to the autonomic computing principles).

The project described in this article has received funding from the French National Agency of Research (ANR) under grant agreement $\mathrm{n}^{\circ}$ ANR-17-CE10-0014-03. 


\section{REFERENCES}

[1] ISO19650-1:2018, "Organization and digitization of information about buildings and civil engineering works, including building information modelling (bim) information management using building information modelling part 1: Concepts and principles," ISO/TC59/SC13, Organization and digitization of information about buildings and civil engineering works, including building information modelling (BIM).

[2] A. Ghosh and T. Givargis, "Lord: a localized, reactive and distributed protocol for node scheduling in wireless sensor networks," in Design, Automation and Test in Europe, March 2005, pp. 190-195 Vol. 1.

[3] H. M. Salman, "Survey of routing protocols in wireless sencor networks," in International Journal of Sensors and Sensor Networks, 2014, pp. 1-6.

[4] S. M. Zin, N. B. Anuar, M. L. M. Kiah, and P. A.-S. K., "Routing protocol design for secure wsn: Review and open research issues," Journal of Network and Computer Application, vol. 41, pp. 517-530, 2014.

[5] A. Anwar and D. Sridharan, "A survey on routing protocols for wireless sensor networks in various environments," International Journal of Computer Applications, vol. 112, no. 5, pp. 13-29, 2015.

[6] P. Singh and S. Singh, "A review: Power efficient gathering in sensor information system (PEGASIS) protocol for WSN," International Research Journal of Engineering and Technology (IRJET, vol. 02, no. 03, pp. 1010-1013, 2015.

[7] W. R. Heinzelman, A. Chandrakasan, and H. Balakrishnan, "Energyefficient communication protocol for wireless microsensor networks," in Proceedings of the 33rd Hawaii International Conference on System Sciences-Volume 8 - Volume 8, ser. HICSS '00. Washington, DC, USA: IEEE Computer Society, 2000, pp. 8020-. [Online]. Available: http://dl.acm.org/citation.cfm?id=820264.820485

[8] S. Lindsey and C. S. Raghavendra, "Pegasis: Power-efficient gathering in sensor information systems," in Proceedings, IEEE Aerospace Conference, vol. 3, March 2002, pp. 3-3.

[9] F. Tang, I. You, S. Guo, M. Guo, and Y. Ma, "A chain-cluster based routing algorithm for wireless sensor networks," Journal of Intelligent Manufacturing, vol. 23, no. 4, pp. 1305-1313, Aug 2012. [Online]. Available: https://doi.org/10.1007/s10845-010-0413-4

[10] Z. Gengsheng and H. Zhengbing, "chain routing based on coordinatesoriented clustering strategy in wsns," in In International Symposium on Computer Network and Multimedia Technology (CNMT), 2009, pp. 1-4.

[11] K. S. Ahn, D. G. Kim, B. S. Sim, H. Y. Youn, and O. Song, "Balanced chain-based routing protocol (bcbrp) for energy efficient wireless sensor networks," in 2011 IEEE Ninth International Symposium on Parallel and Distributed Processing with Applications Workshops, May 2011, pp. 227-231.

[12] W. Derigent, M. David, H. Wan, D. Dragomirescu, A. Takacs, G. Loubet, A.-M. Roxin, R. Melet, and L. Montegut, "Materials communicating wit BIM: aims and first results of the McBIM project," in Proceedings of Structural Health Monitoring, 2019.

[13] ISO29481-1:2016, "Building information models information delivery manual part 1: Methodology and format," ISO/TC59/SC13, Organization and digitization of information about buildings and civil engineering works, including building information modelling (BIM), 2016.

[14] ISO16739-1:2018, "Industry foundation classes (ifc) for data sharing in the construction and facility management industries part 1: Data schema," ISO/TC59/SC13, Organization and digitization of information about buildings and civil engineering works, including building information modelling (BIM), 2018.

[15] ISO10303-21:2016, "Industrial automation systems and integration product data representation and exchange part 21: Implementation methods: Clear text encoding of the exchange structure," ISO/TC59/SC13, Organization and digitization of information about buildings and civil engineering works, including building information modelling (BIM), 2016.

[16] ISO12006-3:2007, "Building construction organization of information about construction works part 3: Framework for object-oriented information," ISO/TC59/SC13, Organization and digitization of information about buildings and civil engineering works, including building information modelling (BIM), 2007.

[17] J. Beetz, "A scalable network of concept libraries using distributed graph databases," in 2014 International Conference on Computing in Civil and Building Engineering, Orlando, Florida, United States, June 2014.
[18] ISO21127:2014, "Information and documentation a reference ontology for the interchange of cultural heritage information," ISO/TC 46/SC 4 Technical interoperability, 2014.

[19] ISO/IEC20944-1:2013, "Information technology metadata registries interoperability and bindings (mdr-ib) part 1: Framework, common vocabulary, and common provisions for conformance," ISO/IEC JTC 1/SC 32 Data management and interchange, 2013.

[20] ISO16678:2014, "Guidelines for interoperable object identification and related authentication systems to deter counterfeiting and illicit trade," ISO/TC 292 Security and resilience, 2014.

[21] ISO/IEC19941:2017, "Information technology cloud computing interoperability and portability," ISO/IEC JTC 1/SC 38 Cloud Computing and Distributed Platforms, 2017.

[22] ISO13606-1:2019, "Health informatics electronic health record communication part 1: Reference model," ISO/TC 215 Health informatics, 2019.

[23] S. Mini, S. K. Udgata, and S. L. Sabat, "Sensor deployment and scheduling for target coverage problem in wireless sensor networks," IEEE Sensors Journal, vol. 14, no. 3, pp. 636-644, 2014.

[24] A. Ndam Njoya, W. Abdou, A. Dipanda, and E. Tonye, "Optimization of sensor deployment using multi-objective evolutionary algorithms," Journal of Reliable Intelligent Environments, vol. 2, no. 4, p. 209220, 2016.

[25] A. Ndam Njoya, C. Thron, J. Barry, Abdou, E. Tonye, N. S. L. Konje, and A. Dipanda, "Efficient scalable sensor node placement algorithm for fixed target coverage applications of wireless sensor networks," IET Wireless Sensor Systems, vol. 7, no. 2, pp. 44-54, 2017.

[26] P. Lalanda, J. A. McCann, and A. Diaconescu, Autonomic Computing Principles, Design and Implementation. The name of the publisher, 2014.

[27] M. C. Huebscher and J. A. McCann, "A survey of autonomic computing\&mdash;degrees, models, and applications," ACM Comput. Surv., vol. 40, no. 3, pp. 7:1-7:28, 2008. 